\title{
A Hybrid Electric Vehicle Transmission Chain Simulator Based on Electromechanical Actuators
}

\author{
Ahmed Chaibet ${ }^{1}$, Cherif Larouci ${ }^{2}$, and Moussa Boukhnifer ${ }^{3}$ \\ ${ }^{1}$ Control and Systems Laboratory,Estaca, ahmed.chaibet@estaca.fr \\ ${ }^{2}$ Control and Systems Laboratory,Estaca,cherif.larouci@estaca.fr \\ ${ }^{3}$ Control and Systems Laboratory, Estaca,moussa.boukhnifer@estaca.fr
}

\begin{abstract}
This paper presents a hybrid electric vehicle transmission chain simulator connected to longitudinal vehicle motion. The transmission simulator uses electric actuators to reproduce the mechanical characteristics of a real vehicle engine and its transmission chain. The developed approach allows to validate transmission and vehicle dynamic studies (control of automatic or robotized gearboxes, test of heat engine, dynamic behavior and passenger comfort...) without need to the real transmission system and the real environment of the vehicle. The proposed system is used to carry out a vehicle automated driving for a cruise control test. The control law strategy consists on a first sliding mode. The performances of the controller are presented to demonstrate the effectiveness of the proposed approach.
\end{abstract}

\section{Keywords}

hybrid electric vehicle, transmission simulator, sliding mode, dynamic, electric actuator

\section{INTRODUCTION}

A lot of works have been developed over the two last decades about the Hybrid electric vehicles (HEVs) [Sciarretta et al., 2004; Bardin, 2000]. These vehicles are powered in many forms depending on the mix of energy sources and propulsion systems used on the vehicle. It can be run on conventional or alternative fuel and an electric motor. The hybrid concept can be used for drives taking energy from two separate energy sources, for series or parallel drive configurations or any combination of them. The most distinct advantages of this concept is to reduce the emission of $\mathrm{CO}_{2}$ and other emissions $(\mathrm{NOx}, \ldots)$ permitted by these vehicles. Moreover the electric motors can be controlled easily and with high precision than that the combustion engines [Hofman and Purnot, 2009]. It is shown that vehicle stability, safety and performances are ensured by controlling the electric motors [Boukhnifer et al., 2011]. In this way simulators and effective methods using accurate models are very useful to reach this objective and to minimize the cost and the time devoted to the development phases of vehicles. In parallel configuration of (HEV) the combustion engine and the electric motor are directly connected to the wheels. In this paper, we will develop an electrical simulator of a parallel HEV configuration by replacing the internal combustion engine part with an equivalent electric actuator. The aim is to propose an accurate equivalent system easy to be exploited to validate simulation studies in the automotive domain without need to the real vehicle and its real environment. Another contribution of this paper is the automated driving by means of a dedicated coupled model (simulator and the longitudinal dynamic motion) for which the vehicle must be able to achieve a whole of autonomous functions. Among these functions: the heading variation, change maneuver and a vehicle follower control system for a single vehicle by maintaining a safety distance [Chaibet et al., 2005; Larouci et al., 2007]. The adopted method is based on the application of the first sliding mode control technique because of the presence of strong nonlinearities in the considered model. Moreover, this control suits well to our application for which the control performances are very depending on the vehicle environmental constraints in particular with respect to the state of the roadway (dry, softened, snow-covered, icy...).

The present paper is organized as follows. In section 2, the vehicle transmission simulator will be addressed and validated by means of simulation and measurement results. In section 3, a vehicle dynamic model will be presented. Section 4 deals with a proposed approach to couple the electric simulator to the vehicle longitudinal dynamic model. In section 5, the coupled model will be applied to the automated driving. The aim of this concept is to increase passenger's safety, to reduce the driver's stress and to improve traffic conditions. Simulation results of the proposed control strategy (cruise control) will be presented. 


\section{THE VEHICLE TRANSMISSION SIMULA- TOR}

The electric simulator of the vehicle transmission chain simulates the mechanical characteristic of the transmission system. This simulator uses two electric actuators controlled with dedicated control laws. The first one reproduces the dynamic driving torque developed by the heat engine and available at the output of the bridge, while the second one simulates the resisting torque due to the whole resisting efforts and inertias.

\subsection{Modeling of the real vehicle transmission system} The Figure 1 illustrates the various forces applied to a vehicle during its motion on a road with an $\alpha$ slope angle. These forces include the driving force and the mean resisting forces.

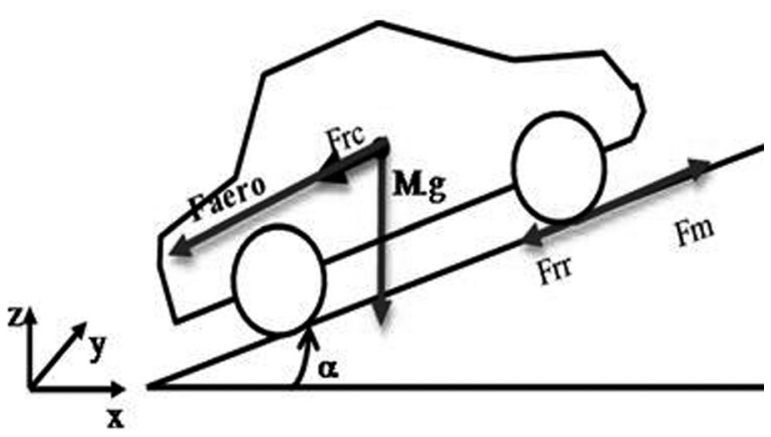

Fig. 1 Forces applied to a vehicle in a slope

$F_{m}$, Faero, $F_{r r}$ and $F_{r c}$ are, respectively, the driving force, the aerodynamics force, the rolling friction force and the resisting force in a slope [BOSCH, 2000; Minakawa et al., 1999].

To model the real vehicle transmission system, we suppose that the transmission losses are neglected (the efficiency of clutch and gear box reaches 1) and only longitudinal forces are considered [Liang et al., 2003; Nakamura et al.,2003; Sawase and Sano, 1999]. According to the centre of gravity of the vehicle and by using these assumptions, the following equations can be written:

$M \cdot \frac{d V}{d t}=F_{m}-F_{\text {aero }}-F_{r r}-F_{r c}$

With:

$\left\{\begin{array}{l}F_{\text {aero }}=\frac{1}{2} \cdot \rho \cdot C_{x} \cdot S_{f} \cdot V^{2}, F_{r r}=f_{r r} \cdot M \cdot g \cdot \cos (\alpha) \\ F_{r c}=M \cdot g \cdot \sin (\alpha), \Omega_{t h}=\Omega_{s p} \cdot R_{t}, V=\Omega_{s p} \cdot R_{s c}\end{array}\right.$

$\Omega_{s p}$ is the angular speed at the output of the bridge. $\Omega_{\text {th }}$ is the angular speed of the heat engine. The transmission is supposed without losses.
So:

$C_{r_{-} \text {roues }}=F_{m} \cdot R_{s c}, C_{m_{-} s p}=C_{r_{-} e b} \cdot R_{t}$

$C_{r_{-} e b}$ is the resisting torque (the resisting torque at the input of the gearbox seen by the heat engine).

From the equation 1, we deduce that:

$\left(J_{s p}+J_{\text {roues }}+M \cdot R_{s c}^{2}\right) \cdot \frac{d \Omega_{s p}}{d t}=C_{m_{-} s p}-C_{s r_{-} s p}$

Where:

$J_{s p}$ and $J_{\text {roues }}$ are, respectively, the inertia at the output of the bridge and the inertia of the wheels.

$C_{s r-s p}$ is the total resisting torque in the steady state at the output of the bridge.

\subsection{Modeling of the equivalent system}

In order to work in a reduced torque scale and to validate the coupling of a transmission model with a vehicle dynamic one, an equivalent model using two electric actuators is considered (Figure 2). In this model, the actuator M2 simulates the whole resisting torque due to aerodynamic frictions, rolling frictions, resisting torque in a slope and inertia with a torque reduction factor ( $\mathrm{fc} 2$ ). However, the electric actuator M1 simulates both the heat engine and the gearbox with a torque reduction factor (fc1), [Chaibet et al., 2008].

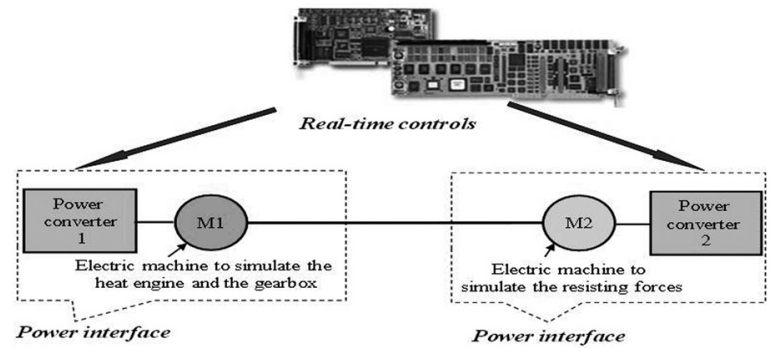

Fig. 2 An equivalent transmission chain model

This model can be used to test control strategies of automatic gearbox and to study the influence of these strategies on the vehicle dynamic behavior in order to improve the passenger comfort for example.

Considering $\mathrm{fc} 2=\mathrm{fc} 1=\mathrm{fc}$ yields:

$\Omega_{1}=\Omega_{2}=\Omega_{s p}=\frac{\Omega_{t h}}{R_{t}}$ and $C_{m_{-} l}=\frac{C_{m-s p}}{f_{C}}$

$\Omega_{1}$ and $\Omega_{2}$ are the angular velocities of the electric actuators M1 and M2. Therefore, the equation 2 can be written as follows:

$\left(J_{\text {sp }}+J_{\text {roues }}\right) \cdot \frac{1}{f_{c}} \cdot \frac{d \Omega_{1}}{d t}=C_{m_{-} 1}-\frac{1}{f_{c}} \cdot C_{r_{-} \text {roues }}$

The mechanical equation on the common tree of the two electric actuators is: 


$$
\left(J_{1}+J_{2}\right) \cdot \frac{d \Omega_{1}}{d t}=C_{m_{-} l}-C_{r_{-} 2}
$$

Where:

$J_{1}$ and $J_{2}$ are the moment of inertia of the actuators M1 and M2. $C_{m_{-} 1}$ and $C_{r_{-} 2}$ are the torques of the actuators $\mathrm{M} 1$ and M2 respectively. An identification between the real model and the equivalent one allows to determine the torques which must be developed by $\mathrm{M} 1$ and M2 to reproduce the behavior of the real transmission chain.

\subsection{Experimental validation of the transmission simulator}

In order to validate the transmission simulator, a measurement test bench is carried out Figure 3.

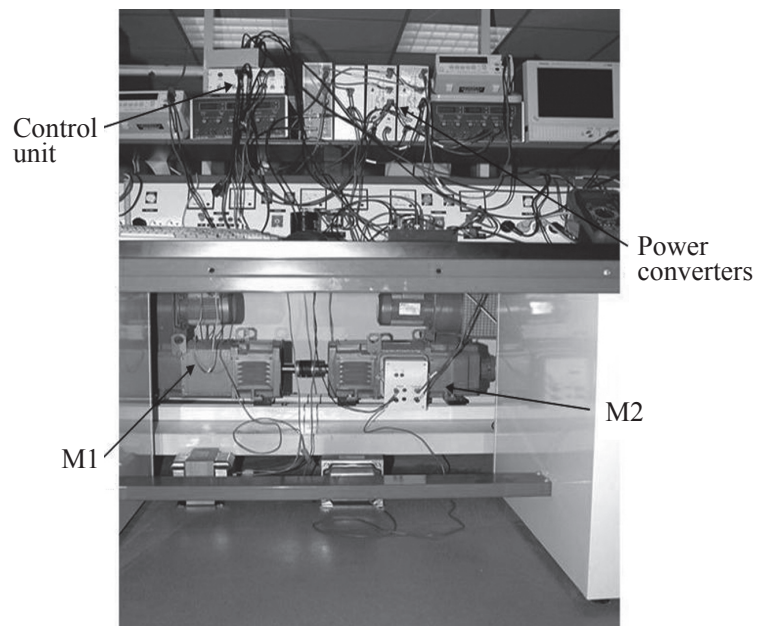

Fig. 3 Measurement test bench

The Figure 4 superimposes the results of simulation (solid lines) and measurement (dashed lines) of the following parameters:

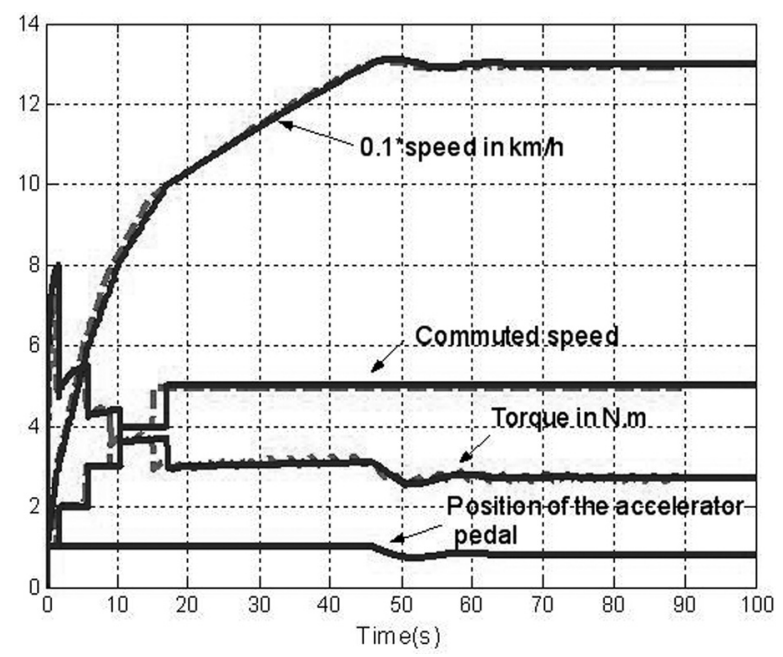

Fig. 4 Measurement (dashed lines) and simulation (solid lines) results (vehicle desired speed $130 \mathrm{~km} / \mathrm{h}$, slope $=0 \%$ )
- The normalized vehicle speed (vehicle speed divided by 10),

- The committed speeds,

- The torque developed by the actuator M1 which simulates the heat engine and the gearbox. This torque is the image of the real torque available at the output of the bridge (with a reduction coefficient $\mathrm{fc}=200$ ),

- The position of the accelerator pedal.

In this case, the roadway is supposed without slope. The vehicle desired speed is $130 \mathrm{~km} / \mathrm{h}$.

It is noted that the simulation results are very close to those obtained by measurement. The vehicle reaches its desired speed after $50 \mathrm{~s}$. The steady state is characterized by a commuted speed: the 5 th one, a reduced torque: $2.8 \mathrm{Nm}$ (real torque: $560 \mathrm{Nm}$ ) and a position of the accelerator pedal: $\mathrm{Pp}=0.8$.

\section{THE VEHICLE DYNAMIC MODEL}

A one degree-of-freedom vehicle motion model is used to validate the coupling approach and to design a longitudinal control in the case of single car following.

In this study, the pitch, the roll, the yaw, the transversal and the vertical dynamics are neglected. Moreover, the longitudinal slip between the tire and the road is not considered.

Under these assumptions, the dynamic behaviour of a vehicle during longitudinal motion can be described by the following equation [Hung et al., 1997]:

$T_{e}-R_{t} \cdot T_{b}+R_{t} \cdot\left(M_{r r}+R s c \cdot C_{x} \cdot V^{2}+M \cdot R s c \cdot \sin (\alpha)\right)=I_{t} \cdot a$

Where:

$I_{t}=\frac{\left(\mathrm{J}_{\mathrm{e}}+\mathrm{R}_{\mathrm{t}}^{2} \cdot\left(\mathrm{J}_{w r}+J_{w f}+M \cdot R s c^{2}\right)\right)}{R_{t} \cdot R s c}:$

Effective vehicle inertia

\section{COUPLING OF THE TRANSMISSION SIM- ULATOR TO THE VEHICLE DYNAMIC MOD- EL}

The coupled model (Figure 5) associates the transmission simulator and the vehicle dynamic model. The engine torque $\left(T_{e}\right)$ of the dynamic model is generated by the transmission simulator presented in section 2 . This coupled model allows to carry out car following concept, various transmission and dynamic behaviour studies and to optimize specific vehicle test benches without need to the real transmission system and the real environment of the vehicle. Moreover, it allows to carry out HIL works and real time control by combining software part (vehicle dynamic model) and hardware part (transmission system) (Figure 5). 


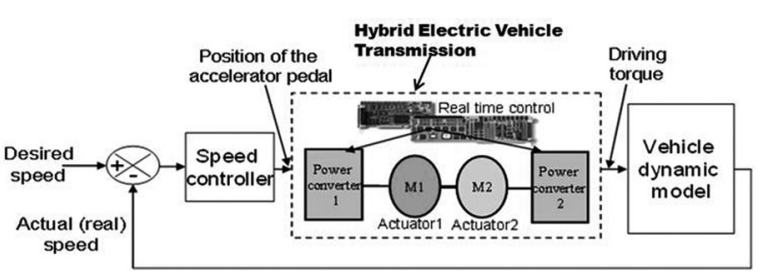

Fig. 5 Block diagram of the coupled model

\section{APPLICATION TO THE VEHICLE AUTO- MATED DRIVING}

On the automated vehicles concept, the vehicle must be able to achieve a whole of autonomous functions. Among these functions, with the respect to longitudinal motion, there are two basic control actions: speed control and spacing control. The whole of this section is dedicated to control strategy for cruise control mode

\subsection{The cruise control mode}

The aim of this part is to design a control low such that the vehicle velocity $v_{f}$ tracks the desired velocity $v_{\text {des }}$. A classical sliding mode control law technique is chosen to track the desired velocity.

\subsubsection{Design methodology}

One considers in this case the sliding manifold is chosen as follows:

$S=v_{f}-v_{d e s}=0$

The derivative of the sliding surface allows to deduce equivalent control $U_{\text {eq }}$ (equations 6 and 7)

$\dot{S}=G+B \cdot U_{e q}=0$

Then, the real control is calculated as follows:

$U=U_{e q}-K_{1} \cdot \operatorname{sign}(S)-K_{2} \cdot S$

$K_{1}$ and $K_{2}$ are control parameters. When the system is far from the sliding manifold, the behavior is dominated by $K_{2}$ term. However, $K_{1}$ term becomes dominant when approaching the manifold. A good choice of $K_{1}$ and $K_{2}$ will allows to reduce both the convergence time and the well-known chattering phenomena near the sliding manifold [Chaibet et al., 2004].

In our case, the derivative of the sliding surface yields:

$\frac{T_{e}-R_{t} \cdot T_{b}+R_{t}\left(M_{r r}+R s c \cdot C_{x} \cdot V^{2}+M \cdot R s c \cdot \sin (\alpha)\right)}{I_{t}}-\dot{v}_{\text {des }}=0$ (9)

The equivalent control input is thus

$U_{e q}=\frac{\left(I_{t} \cdot \dot{v}_{\text {des }}+R_{t} T_{b}-R_{t} \cdot\left(M_{r r}+R s c \cdot C_{x} \cdot V^{2}+M \cdot R s c \cdot \sin (\alpha)\right)\right)}{I_{t}}$

\subsubsection{Simulation Results}

This operation is summarized as follow:

Initially the vehicle evolves at $90 \mathrm{~km} / \mathrm{h}$ on a straight line. The desired velocity $v_{d e s}$ is imposed by the driver (Figure 6).

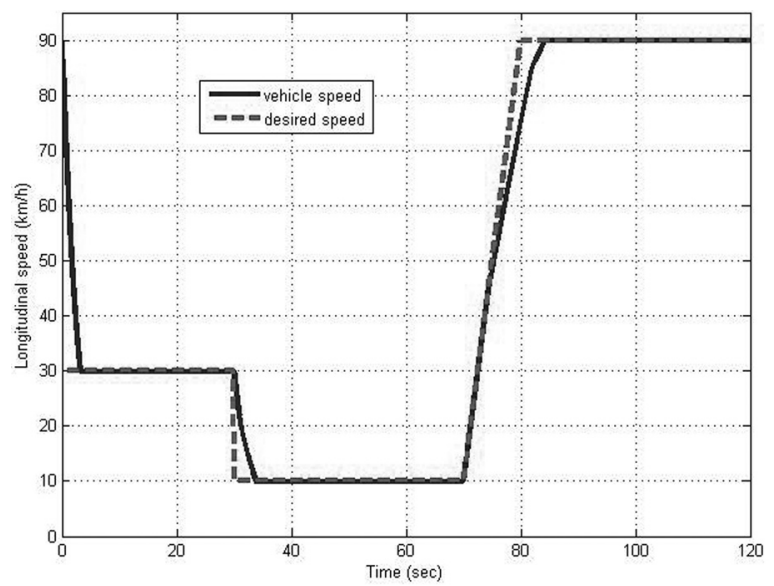

Fig. 6 Longitudinal speeds profile

In order to reach the desired speed, the vehicle decelerates during the first and the second phases and then accelerates during the third one.

Note that the control parameters $K_{1}$ and $K_{2}$ are calculated to ensure good dynamic and static performances (no overshot in the speed and a zero steady state error). The absence of overshot is very important from the safety point of view to respect. Moreover, Figure 7 shows that this operation is carried out with respect to the passenger's comfort [Chaibet et al., 2009].

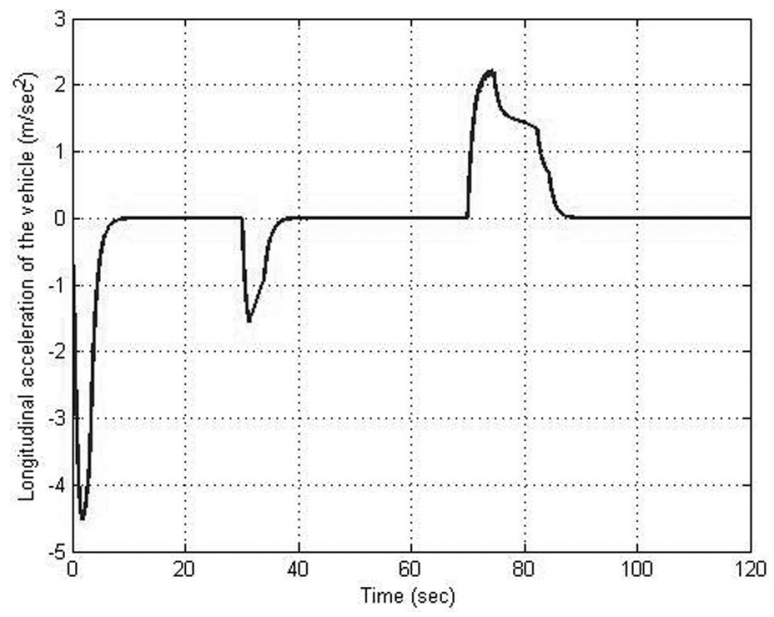

Fig. 7 Longitudinal acceleration

\section{CONCLUSION}

A hybrid electric vehicle transmission simulator coupled to a longitudinal behavior model is developed in this paper and applied to the automated driving control in the case of a cruise control strategy and a carfollowing situation. 
The equivalent transmission system uses electric actuators with dedicated control laws to reproduce the real characteristics of the transmission chain related to the heat engine in a HEV application. The proposed electrical simulator allows validating transmission and vehicle dynamic studies without need to the real transmission system and the real environment of the vehicle.

Due to the presence of strong nonlinearities on the vehicle dynamic model and the parameters uncertainties influenced by the vehicle environment, a sliding mode control strategy is developed. The obtained results demonstrate the ability of the proposed approach to accomplish automated driving by insuring good performances.

\section{References}

Bardin, SIVTHEC, A research program to evaluate high power batteries ageing in actual use, 17th International Battery, Hybrid and Fuel Cell Electric Vehicle Symposium, 2000.

BOSCH, Memento of automotive technology, Bosch Edition, 2000.

Bouknifer, M., A. Chaibet, and C. Larouci, Passivity based control of teleoperated electric vehicle, Journal of Asian Vehicles, Vol. 9, No. 1, 1483-1490, 2011.

Chaibet, A., M. Boukhnifer, and C. Larouci, Estimation for integrated longitudinal and lateral car following control, IEEE MSC-Conference on Control Applications, 1140-1145, 2009.

Chaibet, A., C. Larouci, and E. Grunn, An electric simulator of a vehicle transmission chain coupled to a vehicle dynamic model, Industrial Electronics, IECON, 34th Annual Conference of IEEE, 2008.

Chaibet, A, L. Nouvelière, S. Mammar, and M. Netto, Backstepping control synthesis for both longitudinal and lateral automated low speed vehicle, IEEE Intelligent Vehicles, IV 05, 42-47, 2005.

Chaibet, A., L. Nouvelière, S. Mammar, and M. Netto, Sliding mode control for vehicle following at low speed, IEEE International French Conference on Automatics, 2004.

Hofman, T., and T. Purnot, A comparative study and analysis of an optimized control strategy for the Toyota hybrid system, 24th International Battery, Hybrid and Fuel Cell Electric Vehicle Symposium, 2009.

Hung, P., M. Tomizuka, and K. J. Hedrick, Integrated maneuvering control for automated highway systems based on a magnetic reference/sensing/system, California PATH Research Report, 1997.

Larouci, C., E. Dehondt, A. Harakat, and G. Feld, Modeling and control of the vehicle transmission system using electric actuators; integration of a clutch, IEEE ISIE '07, 2007.

Liang, H., K.T. Chong, T.S. No, and S. Y. Yi, Vehicle longitudinal brake control using variable parameter sliding control, Control Engineering Practice, Vol. 11, No. 4, 403-411, 2003.

Minakawa, M., J. Nakahara, J. Ninomiya, and Y. Rimoto, Method for measuring force transmitted from road surface to tires and its applications, JSAE Review, Vol. 20, No. 4, 479-485, 1999.

Nakamura, K., H. Kosaka, K. Kadota, and K. Shimi$\mathrm{zu}$, Development of a motor assisted 4WD system for small front-wheel-drive vehicles, JSAE Review, Vol. 24, No. 4, 417-424, 2003.

Sawase, K., and Y. Sano, Application of active yaw control to vehicle dynamics by utilizing driving/ breaking force, JSAE Review, Vol. 20, No. 2, 289295, 1999.

Sciarretta, A., M. Back, and L. Guzzella, Optimal control of parallel hybrid electric vehicles, IEEE Transactions on Control Systems Technology, Vol. 12, No. 3, 352-363, 2004.

(Received September 20, 2011; accepted November 17, 2011) 


\section{Appendix}

\begin{tabular}{|c|c|}
\hline$T_{e}$ & engine torque [N.m] \\
\hline$T_{b}$ & brake torque $[\mathrm{N} . \mathrm{m}]$ \\
\hline$M_{r r}$ & rolling resistance torque, $[\mathrm{N} . \mathrm{m}]$ \\
\hline$a$ & longitudinal acceleration $[\mathrm{m} / \mathrm{s} 2]$ \\
\hline$J_{w r}$ and $J_{w f}$ & rear and front wheel inertias $\left[\mathrm{kg} \cdot \mathrm{m}^{2}\right]$ \\
\hline$J_{e}$ & engine / transmission inertia $\left[\mathrm{kg} . \mathrm{m}^{2}\right]$ \\
\hline$C_{x}$ & longitudinal aerodynamic drag coefficient \\
\hline$M$ & the total vehicle mass $[\mathrm{kg}]$ \\
\hline$V$ & the longitudinal vehicle speed $\left[\mathrm{m} / \mathrm{s}^{2}\right]$ \\
\hline$\rho$ & density of the air $\left[\mathrm{kg} / \mathrm{m}^{3}\right]$ \\
\hline$C_{x}$ & the drag coefficient \\
\hline$S_{f}$ & the frontal (transverse) section of the vehicle $\left[\mathrm{m}^{2}\right]$ \\
\hline$f_{r r}$ & the coefficient of rolling friction \\
\hline$g$ & the acceleration of gravity $\left[\mathrm{m} / \mathrm{s}^{2}\right]$ \\
\hline$\alpha$ & the slope angle [rad] \\
\hline$R_{s c}$ & loaded radius (ray of the driving wheel) $[\mathrm{m}]$ \\
\hline$R_{t}=R_{b} R_{p}$ & total reduction ratio \\
\hline$R_{b}$ & the gearbox ratio \\
\hline$R_{p}$ & the bridge ratio \\
\hline
\end{tabular}

